\title{
Envelhecimento acelerado e deterioração controlada em sementes de amendoim
}

\author{
Claudia Antonia Vieira Rossetto(1), Tatiana de Moraes Lima ${ }^{(1)}$ e Eduardo da Costa Guimarães(1) \\ (1)Universidade Federal Rural do Rio de Janeiro, Caixa Postal 74511, CEP 23890-000 Seropédica, RJ. E-mail: cavrosse@ufrrj.br, \\ tmoraes@bol.com.br, ecguimaraes@bol.com.br
}

\begin{abstract}
Resumo - Pesquisas têm sido desenvolvidas visando determinar os procedimentos para a avaliação de diferenças no potencial fisiológico de lotes de sementes, com destaque aos testes de vigor. O objetivo deste trabalho foi estudar o envelhecimento acelerado e a deterioração controlada para avaliação do vigor de sementes de amendoim (Arachis hypogaea). Quatro lotes de sementes de amendoim da cultivar Tatu foram submetidos ao teste de envelhecimento acelerado, em caixas tipo gerbox, com temperatura de $42^{\circ} \mathrm{C}$ ou $43^{\circ} \mathrm{C}$, por 48 horas e 72 horas, com e sem o emprego de solução saturada de $\mathrm{NaCl}$, e ao teste de deterioração controlada, com teor de água de $15 \%$ e $20 \%$, a $40^{\circ} \mathrm{C}$ ou $45^{\circ} \mathrm{C}$, por 48 horas. O teor de água, a germinação e sanidade das sementes foram determinados. Pelos testes de germinação e de emergência, não houve diferença significativa de desempenho entre os quatro lotes. No teste de envelhecimento acelerado com solução salina, o período de 72 horas a $42^{\circ} \mathrm{C}$ é suficiente para avaliar o potencial fisiológico das sementes. No teste de deterioração controlada, a combinação de $15 \%$ de teor de água nas sementes e 48 horas em banho maria a $45^{\circ} \mathrm{C}$ é eficiente para detectar diferenças de vigor entre os lotes.
\end{abstract}

Termos para indexação: Arachis hypogaea, germinação, vigor.

\section{Accelerated aging and controlled deterioration of peanut seeds}

\begin{abstract}
Researches have been developed aiming to determine the procedures for physiological potential seeds evaluation with emphasis to vigour tests. The objective was to study the controlled deterioration and the accelerated aging in peanut (Arachis hypogaea) seeds vigour. Four lots of peanut seeds were submitted to the ageing accelerated test, at $42^{\circ} \mathrm{C}$ or $43^{\circ} \mathrm{C}$, for 48 hours and 72 hours, with or without the use of saturated solution of $\mathrm{NaCl}$, and controlled deterioration test, with $15 \%$ and $20 \%$ water content, at $40^{\circ} \mathrm{C}$ or $45^{\circ} \mathrm{C}$, for 48 hours. The evaluation of water content, germination and health test was accomplished. There was no different performance among the four lots by the germination and emergency tests. In the ageing accelerated test with saturated solution, the period of 72 hours at $42^{\circ} \mathrm{C}$ introduces sensibility to evaluate seeds physiological potential. In the controlled deterioration test, the combination of $15 \%$ of water content, for 48 hours at $45^{\circ} \mathrm{C}$, is efficient to detect vigor differences among peanut seed lots.
\end{abstract}

Index terms: Arachis hypogaea, germination, vigour.

\section{Introdução}

Pesquisas têm procurado determinar os procedimentos para avaliar as diferenças no potencial fisiológico de lotes de sementes. Estudos sobre os testes de vigor estão em evidência, pois há sementes que apresentam porcentagem de germinação semelhante, porém exibem comportamentos distintos no campo e no armazenamento (Marcos Filho, 1999). O teste de envelhecimento acelerado consiste em avaliar a resposta das sementes por meio de teste de germinação, após elas terem sido submetidas à temperatura elevada e umidade relativa do ar próxima a $100 \%$, por determinado período de exposição
(Rossetto \& Marcos Filho, 1995). Em todas as espécies cultivadas, a temperatura e o período de permanência das sementes na câmara não estão estabelecidos e, em amendoim, têm sido indicadas as combinações de $42^{\circ} \mathrm{C}$ por 72 horas (Vanzolini \& Nakagawa, 1998; Rossetto et al., 2003) ou $43^{\circ} \mathrm{C}$ por 48 horas (Usberti \&Amaral, 1999).

Fatores tais como, contaminação inicial das sementes por microorganismos (Silva \& Silva, 2000) e absorção de água pelas sementes (Tekrony, 1993) podem interferir na interpretação dos dados do teste de envelhecimento acelerado. Segundo Powell (1995), a germinação após o período de exposição é inversamente pro- 
porcional ao teor de água. A fim de controlar o processo de embebição e, conseqüentemente, a deterioração, Jianhua \& McDonald (1996) propuseram o uso da solução saturada de sais, em substituição à água destilada durante a realização do teste, permitindo reduzir a umidade relativa do ambiente no interior dos compartimentos individuais e, com isso retardar a absorção de água pelas sementes. Quanto à contaminação por fungos, a alta incidência de Aspergillus spp. e de Rhizopus spp. limita a estimativa do vigor das sementes de amendoim (Freitas, 2000), pois estes microrganismos influenciam na deterioração das sementes durante o teste (Rossetto et al., 2003). No entanto, o controle do desenvolvimento de fungos pode ser realizado com o uso de solução saturada de sais (Jianhua \& McDonald, 1996; Rodo et al., 2000; Panobianco \& Marcos Filho, 2001).

$\mathrm{O}$ teste de deterioração controlada também foi desenvolvido como um teste de vigor, com maior precisão no controle da umidade relativa do ar, durante o envelhecimento (Rodo et al., 2000). No entanto, Tekrony (1993) considerou que o teor de água inicial das sementes deve ser de $15,5 \%$ (base úmida) e, caso o teor de água não seja esse, sugere que ele deva ser atingido pelo processo de embebição controlada. Comparando os testes de envelhecimento acelerado e o de deterioração controlada em sementes de soja, Rossetto \& Marcos Filho (1995) e Marcos Filho et al. (2001) constataram alta correlação entre os testes, revelando as diferenças entre os lotes. Segundo Rossetto \& Marcos Filho (1995), o teste de envelhecimento acelerado é mais drástico do que o de deterioração controlada, pois neste o teor de água das sementes mantém-se constante, e no de envelhecimento acelerado, o teor de água aumenta durante o período de exposição, ocorrendo a diferentes velocidades.

O objetivo deste trabalho foi estudar o envelhecimento acelerado e a deterioração controlada para avaliação do vigor de sementes de amendoim.

\section{Material e Métodos}

O trabalho foi realizado no Laboratório de Análise de Sementes da Universidade Federal Rural do Rio de Janeiro, em 2003. Foram utilizadas amostras de quatro lotes de sementes de amendoim da cultivar Tatu, cedidos pela COPLANA e provenientes de Ribeirão Preto, SP (lote 1), Votuporanga, SP (lote 2), Adamantina, SP (lote 3) e Jaboticabal, SP (lote 4). As sementes foram acondicionadas em sacos de papel Kraft e armazena- das em ambiente a $18^{\circ} \mathrm{C}$ e $45 \%$ de umidade relativa do ar, permanecendo nestas condições até o final da fase experimental. As amostras de cada lote foram submetidas à determinação do teor de água, empregando quatro subamostras de 25 sementes, utilizando o método da estufa, a $105 \pm 3^{\circ} \mathrm{C}$, durante 24 horas, de acordo com Brasil (1992) e, posteriormente, foram caracterizadas quanto à qualidade sanitária e fisiológica inicial.

O teste de sanidade foi realizado com quatro subamostras de 50 sementes, previamente tratadas com hipoclorito de sódio a $2 \%$ por cinco minutos, que foram distribuídas em cinco placas de Petri contendo o meio BDA acrescido de $\mathrm{NaCl}(6 \%)$ e mantidas a $20^{\circ} \mathrm{C}$, em regime de 12 horas de luz, por cinco dias (Ito et al., 1992). Após a incubação, foi realizada a avaliação dos fungos presentes nas sementes, com auxílio de microscópio estereoscópico. Considerou-se a incidência de Rhizopus ssp., de Fusarium spp. e de Aspergillus spp. (Singh et al., 1991). Os resultados foram expressos em porcentagem de sementes infectadas por fungos.

$\mathrm{O}$ teste de germinação foi realizado com quatro subamostras de 50 sementes distribuídas em rolos de papel toalha tipo germitest embebidos com água destilada e mantidas a $25^{\circ} \mathrm{C}$. As avaliações foram realizadas no quinto e décimo dia da instalação do teste, adotandose os critérios estabelecidos nas Regras para Análise de Sementes (Brasil, 1992). Simultaneamente ao teste de germinação, foi realizado o teste de primeira contagem, sendo a porcentagem de plântulas normais obtida no quinto dia após a instalação.

O teste de condutividade elétrica foi realizado com quatro subamostras de 50 sementes, previamente pesadas, imersas em $75 \mathrm{~mL}$ de água destilada durante 24 horas e mantidas a $20^{\circ} \mathrm{C}$, de acordo com Vanzolini \& Nakagawa (1998). Após este período, foi realizada a leitura da condutividade elétrica da solução de embebição, com condutivímetro digital. Os resultados foram divididos pela massa de sementes e a condutividade elétrica expressa em $\mu \mathrm{S} \mathrm{cm}^{-1} \mathrm{~g}^{-1}$.

$O$ teste de emergência foi realizado com quatro subamostras de 50 sementes distribuídas em bandejas de plástico contendo areia, com base em Nakagawa (1999). A contagem das plântulas emersas foi realizada aos dez dias após a semeadura, e os resultados foram expressos em porcentagem.

No teste de envelhecimento acelerado, quatro subamostras de 150 sementes foram distribuídas numa camada única e uniforme, sobre tela de alumínio fixada em caixa de plástico (gerbox), contendo no fundo $40 \mathrm{~mL}$ 
de água destilada ou de solução de $\mathrm{NaCl}$ (40\%), de acordo com Jianhua \& McDonald (1996) e Rodo et al. (2000). As caixas foram mantidas a $42^{\circ} \mathrm{C}$ ou $43^{\circ} \mathrm{C}$, por 48 horas e 72 horas. Posteriormente foram instalados os testes de teor de água e de germinação (Brasil, 1992), visando calcular, respectivamente, o teor de água após a saída das sementes da câmara de envelhecimento e a porcentagem de plântulas normais no quinto e no décimo dia. Conjuntamente, foi realizado o teste de sanidade, que consistiu na identificação de patógenos e seus sintomas, avaliando-se as estruturas nas sementes e nas plântulas, sob microscópios (Brasil, 1992), sendo que a análise da freqüência dos fungos foi realizada no décimo dia após a instalação, com base em Rossetto et al. (2003). Todos os resultados foram expressos em porcentagem.

No teste de deterioração controlada, primeiramente as amostras de sementes de cada lote foram subdivididas em duas subamostras, visando ajustar o teor de água para $15 \%$ e $20 \%$, através de umedecimento pelo método da atmosfera úmida (Rossetto et al., 1995). Para isto, 300 sementes por subamostra foram distribuídas em bandeja de plástico contendo seis folhas de papel germitest umedecido com água destilada, na proporção de 2,5 vezes o peso do papel e mantidas a $10^{\circ} \mathrm{C}$, até atingir o teor de água desejado. Após o umedecimento, as sementes foram acondicionadas em recipientes de vidro, a $10^{\circ} \mathrm{C}$, por sete dias, para atingir o equilíbrio higroscópico. Posteriormente, as sementes de cada subamostra foram acondicionadas em sacos de papelalumínio vedados, imersos em água a $40^{\circ} \mathrm{C}$ ou $45^{\circ} \mathrm{C}$, em aparelho tipo banho-maria, por 48 horas (Rossetto \& Marcos Filho, 1995). Após a exposição, os recipientes foram retirados e mergulhados em água fria por $30 \mathrm{mi}-$ nutos. Em seguida foram instalados os testes de teor de água e de germinação (Brasil, 1992), visando calcular, respectivamente, o teor de água após a exposição das sementes e a porcentagem de plântulas normais no quinto e, também, no décimo dia. Paralelamente, foi realizado o teste de sanidade, com base nos procedimentos descritos para o teste de envelhecimento acelerado, sendo os resultados expressos em porcentagem.

O delineamento experimental foi inteiramente casualizado, com quatro repetições. Os dados em porcentagem foram transformados previamente em arco seno da raiz quadrada de $\mathrm{x} / 100$, embora nas tabelas encontrem-se os dados originais. As médias foram comparadas entre si pelo teste de Tukey a 5\% de probabilidade. Realizou-se a análise de correlação linear simples entre os resultados de deterioração controlada em relação aos de envelhecimento acelerado e da condutividade elétrica.

\section{Resultados e Discussão}

Os dados referentes ao teor de água inicial das sementes foram semelhantes nos quatro lotes (Tabela 1). Para Panobianco \& Marcos Filho (2001), este fato é importante na execução dos testes, uma vez que a uniformização do teor de água das sementes é fundamental para a padronização das avaliações e obtenção de resultados consistentes.

Pelos testes de germinação e de emergência, podese constatar que não houve diferença de desempenho entre os quatro lotes (Tabela 1). As sementes do lote 2 apresentaram maior população de Rhizopus spp., embora este valor não tenha diferido dos apresentados pelos lotes 1 e 3. Em sementes de feijão, Novembre \& Marcos Filho (1991) observaram resultados que indicam a contribuição de Rhizopus spp. na redução da germinação das sementes. Considerando $70 \%$ como valor mínimo de germinação aceitável para a semeadura do amendoim e o estabelecimento adequado de plantas no campo (Comissão Estadual de Sementes e Mudas, 1999), foi constatado que as sementes dos quatro lotes poderiam ser comercializadas.

Tabela 1. Dados médios de teor de água, de condutividade elétrica, de plântulas normais na primeira contagem, de germinação, de emergência e de fungos, obtidos de quatro lotes de sementes de amendoim, por ocasião da avaliação inicial ${ }^{(1)}$.

\begin{tabular}{ccccccccc}
\hline Lotes & $\begin{array}{c}\text { Teor de } \\
\text { água }(\%)\end{array}$ & $\begin{array}{c}\text { Condutividade } \\
\text { elétrica }\left(\mu \mathrm{S} \mathrm{m}^{-1} \mathrm{~g}^{-1}\right)\end{array}$ & $\begin{array}{c}\text { Primeira } \\
\text { contagem }(\%)\end{array}$ & $\begin{array}{c}\text { Germinação } \\
(\%)\end{array}$ & $\begin{array}{c}\text { Emergência } \\
(\%)\end{array}$ & $\begin{array}{c}\text { Rhizopus } \\
\text { spp. }(\%)\end{array}$ & $\begin{array}{c}\text { Fusarium spp. Aspergillus spp. } \\
(\%)\end{array}$ & $\begin{array}{c}\text { (\%) } \\
(\%)\end{array}$ \\
\hline 1 & $5,6 \mathrm{a}$ & $222,3 \mathrm{ab}$ & $42,0 \mathrm{a}$ & $87,0 \mathrm{a}$ & $59,0 \mathrm{a}$ & $11,75 \mathrm{ab}$ & $0,50 \mathrm{a}$ & $0,50 \mathrm{a}$ \\
2 & $5,4 \mathrm{a}$ & $162,1 \mathrm{~b}$ & $37,0 \mathrm{a}$ & $86,0 \mathrm{a}$ & $38,0 \mathrm{a}$ & $28,75 \mathrm{a}$ & $4,00 \mathrm{a}$ & $0,25 \mathrm{a}$ \\
3 & $5,2 \mathrm{a}$ & $305,3 \mathrm{a}$ & $16,0 \mathrm{a}$ & $75,0 \mathrm{a}$ & $27,0 \mathrm{a}$ & $4,00 \mathrm{~b}$ & $1,00 \mathrm{a}$ & $4,50 \mathrm{a}$ \\
4 & $5,4 \mathrm{a}$ & $228,0 \mathrm{ab}$ & $33,0 \mathrm{a}$ & $83,0 \mathrm{a}$ & $55,0 \mathrm{a}$ & $21,50 \mathrm{ab}$ & $5,25 \mathrm{a}$ & $0,00 \mathrm{a}$ \\
\hline
\end{tabular}

${ }^{(1)}$ Médias seguidas pela mesma letra na coluna não diferem entre si pelo teste de Tukey a $5 \%$ de probabilidade. 
A primeira contagem de germinação também não revelou diferença significativa entre os quatro lotes (Tabela 1). No entanto, pelo teste de condutividade elétrica, constatou-se que o lote 2 apresentou menor lixiviação de exsudatos para a solução de embebição, evidenciando mais baixo valor de condutividade (Tabela 1). Segundo Tilden \& West (1985), um menor valor de condutividade indica maior rapidez na organização do sistema de membranas celulares e, conseqüentemente, maior vigor. Além disso, por este teste, pode-se destacar outros dois níveis de potencial fisiológico, sendo que os lotes 1 e 4 apresentaram vigor intermediário e o lote 3 apresentou menor vigor.

No teste de envelhecimento acelerado, quando as sementes dos quatro lotes foram expostas por 72 horas a $42^{\circ} \mathrm{C}$, com ou sem o uso de solução saturada de sal, e avaliadas ao quinto e décimo dia da instalação, foi constatada a qualidade superior do lote 2 e o pior desempenho do lote 3 , e foram identificados os lotes 1 e 4 como de nível intermediário (Tabela 2). Estes resultados concordam com a classificação dos lotes pelo teste de condutividade elétrica (Tabela 1). Ao submeter as sementes por 48 horas a $43^{\circ} \mathrm{C}$, com ou sem solução salina, foi detectada, nas avaliações realizadas no quinto e décimo dia, a melhor performance das sementes do lote 1 , e foram identificados os lotes 2 e 4 como de nível inter- mediário. Comparando-se a amplitude da variação entre os dados de temperatura e de período de exposição, os valores de porcentagem de plântulas normais obtidos nas avaliações realizadas no quinto e décimo dia foram maiores, considerando-se o período de exposição, do que os da temperatura (Tabela 2). Bhering et al. (2003), trabalhando com sementes de melancia, observaram que o período de exposição teve efeito mais pronunciado na eficiência do teste para avaliação do vigor das sementes do que a temperatura. Em relação ao teor de água das sementes dos quatro lotes, foi constatado que quando não foi utilizada a solução salina, a variação do teor de água foi de 1,4 a 2,7\% (Tabela 3). De acordo com Marcos Filho (1999), somente valores acima de 3\% a $4 \%$ no teor de água das amostras de sementes comprometem os resultados deste teste, uma vez que condicionam variação na velocidade de umedecimento durante o envelhecimento e, consequientemente, diferenças na velocidade de deterioração. Além disso, neste sistema, o teor de água atingiu valores de 19\% a 26\%. Estes valores foram semelhantes aos encontrados em sementes de amendoim com teor inicial de 7,2\% de água, quando foi realizado o teste a $42^{\circ} \mathrm{C}$ por 72 horas (Rossetto et al., 2003).

Verificou-se que, quando as sementes foram expostas à solução saturada de sal, elas apresentaram valo-

Tabela 2. Dados médios, em porcentagem, de plântulas normais, obtidos de quatro lotes de sementes de amendoim, por ocasião da exposição ao envelhecimento acelerado e à deterioração controlada ${ }^{(1)}$.

\begin{tabular}{|c|c|c|c|c|c|c|c|c|c|c|c|c|c|}
\hline \multirow[t]{3}{*}{ Avaliações } & \multirow[t]{3}{*}{ Lotes } & \multicolumn{4}{|c|}{$\begin{array}{l}\text { Envelhecimento acelerado - } \\
\text { Com solução salina }\end{array}$} & \multicolumn{4}{|c|}{$\begin{array}{l}\text { Envelhecimento acelerado - } \\
\text { Sem solução salina }\end{array}$} & \multicolumn{4}{|c|}{$\begin{array}{c}\text { Deterioração } \\
\text { controlada }\end{array}$} \\
\hline & & \multicolumn{2}{|c|}{$42^{\circ} \mathrm{C}$} & \multicolumn{2}{|c|}{$43^{\circ} \mathrm{C}$} & \multicolumn{2}{|c|}{$42^{\circ} \mathrm{C}$} & \multicolumn{2}{|c|}{$43^{\circ} \mathrm{C}$} & \multicolumn{2}{|c|}{$40^{\circ} \mathrm{C}$} & \multicolumn{2}{|c|}{$45^{\circ} \mathrm{C}$} \\
\hline & & $48 \mathrm{~h}$ & $72 \mathrm{~h}$ & $48 \mathrm{~h}$ & $72 \mathrm{~h}$ & $48 \mathrm{~h}$ & $72 \mathrm{~h}$ & $48 \mathrm{~h}$ & $72 h$ & $15 \%$ & $20 \%$ & $15 \%$ & $20 \%$ \\
\hline \multirow[t]{4}{*}{5 dias } & 1 & $45,0 \mathrm{a}$ & $40,0 \mathrm{~b}$ & $53,0 \mathrm{a}$ & $21,0 \mathrm{a}$ & $46,0 \mathrm{a}$ & $36,0 \mathrm{ab}$ & $41,0 \mathrm{a}$ & $13,0 \mathrm{a}$ & $25,0 \mathrm{a}$ & $21,0 \mathrm{a}$ & $26,0 \mathrm{ab}$ & $25,0 \mathrm{a}$ \\
\hline & 2 & $39,0 \mathrm{a}$ & $67,0 \mathrm{a}$ & $29,0 \mathrm{ab}$ & $18,0 \mathrm{a}$ & $30,0 \mathrm{a}$ & $57,0 \mathrm{a}$ & $36,0 \mathrm{ab}$ & $14,0 \mathrm{a}$ & $20,0 \mathrm{ab}$ & $36,0 \mathrm{a}$ & $40,0 \mathrm{a}$ & $12,0 \mathrm{a}$ \\
\hline & 3 & $16,0 \mathrm{~b}$ & $19,0 \mathrm{c}$ & $17,0 \mathrm{~b}$ & $13,0 \mathrm{a}$ & $13,0 \mathrm{~b}$ & $15,0 \mathrm{c}$ & $13,0 \mathrm{~b}$ & $11,0 \mathrm{a}$ & $3,0 \mathrm{~b}$ & $10,0 \mathrm{~b}$ & $10,0 \mathrm{~b}$ & $2,0 \mathrm{~b}$ \\
\hline & 4 & $20,0 \mathrm{ab}$ & $38,0 \mathrm{~b}$ & $25,0 \mathrm{ab}$ & $10,0 \mathrm{a}$ & $37,0 \mathrm{a}$ & $35,0 \mathrm{~b}$ & $38,0 \mathrm{ab}$ & $9,0 \mathrm{a}$ & $16,0 \mathrm{ab}$ & $27,0 \mathrm{a}$ & $25,0 \mathrm{ab}$ & $20,0 \mathrm{a}$ \\
\hline \multirow[t]{4}{*}{10 dias } & 1 & $81,0 \mathrm{a}$ & $68,0 \mathrm{ab}$ & $87,0 \mathrm{a}$ & $73,0 \mathrm{a}$ & $69,0 \mathrm{a}$ & $65,0 \mathrm{ab}$ & $81,0 \mathrm{a}$ & $60,0 \mathrm{a}$ & $86,0 \mathrm{a}$ & $58,0 \mathrm{a}$ & $82,0 \mathrm{ab}$ & $85,0 \mathrm{a}$ \\
\hline & 2 & $85,0 \mathrm{a}$ & $85,0 \mathrm{a}$ & $72,0 \mathrm{ab}$ & $71,0 \mathrm{a}$ & $71,0 \mathrm{a}$ & $81,0 \mathrm{a}$ & $63,0 \mathrm{ab}$ & $64,0 \mathrm{a}$ & $71,0 \mathrm{ab}$ & $51,0 \mathrm{a}$ & $86,0 \mathrm{a}$ & $41,0 \mathrm{~b}$ \\
\hline & 3 & $62,0 \mathrm{a}$ & $59,0 \mathrm{~b}$ & $69,0 \mathrm{~b}$ & $58,0 \mathrm{a}$ & $60,0 \mathrm{a}$ & $58,0 \mathrm{~b}$ & $56,0 \mathrm{~b}$ & $50,0 \mathrm{a}$ & $66,0 \mathrm{~b}$ & $69,0 \mathrm{a}$ & $56,0 \mathrm{~b}$ & $62,0 \mathrm{a}$ \\
\hline & 4 & $66,0 \mathrm{a}$ & $68,0 \mathrm{ab}$ & $74,0 \mathrm{ab}$ & $65,0 \mathrm{a}$ & $61,0 \mathrm{a}$ & $65,0 \mathrm{ab}$ & $70,0 \mathrm{ab}$ & $58,0 \mathrm{a}$ & $44,0 \mathrm{~b}$ & $69,0 \mathrm{a}$ & $72,0 \mathrm{ab}$ & $68,0 \mathrm{a}$ \\
\hline
\end{tabular}

${ }^{(1)}$ Médias seguidas pela mesma letra na coluna não diferem entre si pelo teste de Tukey a $5 \%$ de probabilidade.

Tabela 3. Dados médios, em porcentagem, de teor de água, obtidos de quatro lotes de sementes de amendoim, por ocasião da exposição ao envelhecimento acelerado e à deterioração controlada.

\begin{tabular}{|c|c|c|c|c|c|c|c|c|c|c|c|c|}
\hline \multirow[t]{3}{*}{ Lotes } & \multicolumn{4}{|c|}{$\begin{array}{l}\text { Envelhecimento acelerado - } \\
\text { Com solução salina }\end{array}$} & \multicolumn{4}{|c|}{$\begin{array}{c}\text { Envelhecimento acelerado - } \\
\text { Sem solução salina }\end{array}$} & \multicolumn{4}{|c|}{$\begin{array}{c}\text { Deterioração } \\
\text { controlada }\end{array}$} \\
\hline & \multicolumn{2}{|c|}{$42^{\circ} \mathrm{C}$} & \multicolumn{2}{|c|}{$43^{\circ} \mathrm{C}$} & \multicolumn{2}{|c|}{$42^{\circ} \mathrm{C}$} & \multicolumn{2}{|c|}{$43^{\circ} \mathrm{C}$} & \multicolumn{2}{|c|}{$40^{\circ} \mathrm{C}$} & \multicolumn{2}{|c|}{$45^{\circ} \mathrm{C}$} \\
\hline & $48 \mathrm{~h}$ & $72 \mathrm{~h}$ & $48 \mathrm{~h}$ & $72 \mathrm{~h}$ & $48 \mathrm{~h}$ & $72 \mathrm{~h}$ & $48 \mathrm{~h}$ & $72 \mathrm{~h}$ & $15 \%$ & $20 \%$ & $15 \%$ & $20 \%$ \\
\hline 1 & 8,7 & 8,3 & 7,9 & 8,3 & 21,2 & 24,2 & 20,9 & 24,7 & 16,7 & 19,9 & 15,0 & 19,8 \\
\hline 2 & 7,8 & 8,2 & 7,9 & 8,2 & 20,9 & 24,3 & 20,6 & 24,8 & 15,1 & 20,4 & 16,0 & 19,1 \\
\hline 3 & 7,7 & 9,0 & 7,9 & 7,7 & 21,1 & 21,6 & 20,0 & 22,6 & 15,2 & 20,8 & 15,4 & 19,4 \\
\hline 4 & 7,7 & 8,3 & 7,9 & 7,9 & 22,3 & 22,0 & 19,7 & 24,1 & 15,0 & 20,8 & 16,4 & 19,8 \\
\hline
\end{tabular}


res uniformes de teor de água após o envelhecimento acelerado e inferiores aos constatados pelas sementes que não foram expostas à solução salina (Tabela 3). Para Rodo et al. (2000) e Panobianco \& Marcos Filho (2001), esse menor teor de água causa prejuízos menores na germinação quando comparado ao uso do método que não emprega a solução salina, indicando menor taxa de deterioração sob esta condição. Pela análise de freqüência dos fungos nas plântulas deterioradas e nas sementes mortas, constatou-se também, que as sementes do lote 2 , quando expostas a $42^{\circ} \mathrm{C}$ por 72 horas, usando solução salina, apresentaram menor porcentagem de fungos do gênero Rhizopus, do que as dos lotes 1 e 4, embora este valor não tenha diferido do apresentado pelas sementes do lote 3 (Tabela 4). Jianhua \& McDonald (1996), Rodo et al. (2000) e Panobianco \& Marcos Filho (2001) constataram que a baixa umidade relativa do ar promovida pela adição de solução saturada de sais impede o crescimento de microrganismos, minimizando a preocupação com o efeito de patógenos associados às sementes sobre os resultados do teste de envelhecimento acelerado.

Examinando os dados obtidos no teste de deterioração controlada (Tabela 2), observou-se, que quando as sementes dos quatro lotes, com $15 \%$ de água, foram expostas a $45^{\circ} \mathrm{C}$, e avaliadas ao quinto e décimo dia da instalação, foi constatada ordenação dos lotes semelhante à obtida pelo teste de condutividade elétrica (Tabela 1) e pelo teste de envelhecimento acelerado a $42^{\circ} \mathrm{C}$, por 72 horas (Tabela 2). Assim, o lote 2 foi classificado como superior, o lote 3 como inferior e os lotes 1 e 4 como de qualidade intermediária. Ao submeter as sementes com $15 \%$ de água a $40^{\circ} \mathrm{C}$, foi verificado, nas avaliações ao quinto e décimo dia, que o lote 2 apresentou comportamento inferior ao lote 1 , assim como constatado pelo teste de envelhecimento acelerado a $43^{\circ} \mathrm{C}$, por 48 horas (Tabela 2). Padilha et al. (2001), avaliando o desempenho de sementes de milho pelo teste de deterioração controlada, constataram que sementes com maior vigor apresentam desempenho superior em uma faixa mais ampla de condições ambientais. Sendo assim, lotes com menor qualidade, como o lote 1, revelaram maior dificuldade de resistir às condições de estresse. Verificou-se que quando foi realizado o teste de deterioração controlada, com sementes apresentando $20 \%$ de água, não houve separação entre os lotes em três níveis de vigor (Tabela 2). Estes resultados concordam com os de Rossetto \& Marcos Filho (1995), os quais observaram, em sementes de soja, que, quanto maior o teor de água das sementes, maior o efeito deletério. Com relação aos dados referentes ao teor de água após o teste de deterioração controlada (Tabela 3), foi observado que as sementes permaneceram com teores de água semelhantes aos do início do teste. Estes resultados concordam com as afirmações de Rossetto \& Marcos Filho (1995) que relatam que esse teste permite o controle do teor de água das sementes durante a deterioração. A análise da freqüência dos fungos nas plântulas deterioradas e nas sementes mortas revela que as sementes do lote 2 , com $15 \%$, quando expostas a $45^{\circ} \mathrm{C}$, apresentaram menor porcentagem de Rhizopus spp. do que as dos lotes 1 e 4, embora este não tenha diferido do valor das sementes do lote 3 (Tabela 5).

Os resultados do teste de deterioração controlada realizado no quinto e décimo dia, utilizando sementes com teor inicial de água de $15 \%$ sob temperatura de $45^{\circ} \mathrm{C}$, apresentaram coeficientes de correlação elevados e significativos (Tabela 6), quando comparados aos resultados do teste de envelhecimento acelerado com solução salina, a $42^{\circ} \mathrm{C}$ por 72 horas, obtidos nas avaliações no quinto e décimo dia, assim como com os resultados do teste de condutividade elétrica, indicando que houve associação entre esses testes. Santos et al. (2003),

Tabela 4. Dados médios, em porcentagem, de fungos encontrados nas sementes e nas plântulas de amendoim provenientes de quatro lotes, por ocasião da exposição ao teste de envelhecimento acelerado $^{(1)}$.

\begin{tabular}{|c|c|c|c|c|c|c|c|}
\hline \multirow{3}{*}{$\begin{array}{l}\text { Tempe- } \\
\text { ratura/ } \\
\text { período }\end{array}$} & \multirow[t]{3}{*}{ Lotes } & \multicolumn{2}{|c|}{$\begin{array}{c}\text { Rhizopus } \\
\text { spp. }\end{array}$} & \multicolumn{2}{|c|}{$\begin{array}{c}\text { Fusarium } \\
\text { spp. }\end{array}$} & \multicolumn{2}{|c|}{$\begin{array}{c}\text { Aspergillus } \\
\text { spp. }\end{array}$} \\
\hline & & Com & Sem & Com & Sem & Com & Sem \\
\hline & & $\begin{array}{c}\text { solução } \\
\text { salina }\end{array}$ & $\begin{array}{c}\text { solução } \\
\text { salina }\end{array}$ & $\begin{array}{c}\text { solução } \\
\text { salina }\end{array}$ & $\begin{array}{c}\text { solução } \\
\text { salina }\end{array}$ & $\begin{array}{c}\text { solução } \\
\text { salina }\end{array}$ & $\begin{array}{c}\text { solução } \\
\text { salina }\end{array}$ \\
\hline \multirow[t]{4}{*}{$42^{\circ} \mathrm{C} / 48 \mathrm{~h}$} & 1 & $2,0 \mathrm{~b}$ & $23,0 \mathrm{a}$ & $2,0 \mathrm{a}$ & $1,0 \mathrm{~b}$ & $0,0 \mathrm{a}$ & $1,0 \mathrm{a}$ \\
\hline & 2 & $6,0 \mathrm{ab}$ & $26,0 \mathrm{a}$ & $1,0 \mathrm{a}$ & $0,0 \mathrm{~b}$ & $0,0 \mathrm{a}$ & $0,0 \mathrm{a}$ \\
\hline & 3 & $0,0 \mathrm{~b}$ & $7,0 \mathrm{~b}$ & $0,0 \mathrm{a}$ & $9,0 \mathrm{a}$ & $2,0 \mathrm{a}$ & $0,0 \mathrm{a}$ \\
\hline & 4 & $10,0 \mathrm{a}$ & $19,0 \mathrm{a}$ & $3,0 \mathrm{a}$ & $0,0 \mathrm{~b}$ & $0,0 \mathrm{a}$ & $0,0 \mathrm{a}$ \\
\hline \multirow[t]{4}{*}{$42^{\circ} \mathrm{C} / 72 \mathrm{~h}$} & 1 & $21,0 \mathrm{a}$ & $23,0 \mathrm{a}$ & $4,0 \mathrm{a}$ & $4,0 \mathrm{a}$ & $0,0 \mathrm{a}$ & $0,0 \mathrm{a}$ \\
\hline & 2 & $6,0 b c$ & $18,0 \mathrm{a}$ & $3,0 \mathrm{a}$ & $1,0 \mathrm{a}$ & $0,0 \mathrm{a}$ & $0,0 \mathrm{a}$ \\
\hline & 3 & $2,0 \mathrm{c}$ & $23,0 \mathrm{a}$ & $2,0 \mathrm{a}$ & $5,0 \mathrm{a}$ & $0,0 \mathrm{a}$ & $0,0 \mathrm{a}$ \\
\hline & 4 & $17,0 \mathrm{ab}$ & $27,0 \mathrm{a}$ & $1,0 \mathrm{a}$ & $1,0 \mathrm{a}$ & $0,0 \mathrm{a}$ & $0,0 \mathrm{a}$ \\
\hline \multirow[t]{4}{*}{$43^{\circ} \mathrm{C} / 48 \mathrm{~h}$} & 1 & $4,0 \mathrm{~b}$ & $14,0 \mathrm{a}$ & $0,0 \mathrm{a}$ & $2,0 \mathrm{a}$ & $0,0 \mathrm{a}$ & $0,0 \mathrm{~b}$ \\
\hline & 2 & $16,0 \mathrm{a}$ & $18,0 \mathrm{a}$ & $2,0 \mathrm{a}$ & $0,0 \mathrm{a}$ & $1,0 \mathrm{a}$ & $3,0 \mathrm{a}$ \\
\hline & 3 & $1,0 \mathrm{~b}$ & $0,0 \mathrm{~b}$ & $0,0 \mathrm{a}$ & $0,0 \mathrm{a}$ & $0,0 \mathrm{a}$ & $2,0 \mathrm{a}$ \\
\hline & 4 & $11,0 \mathrm{a}$ & 7,0ab & $1,0 \mathrm{a}$ & $2,0 \mathrm{a}$ & $0,0 \mathrm{a}$ & $0,0 \mathrm{~b}$ \\
\hline \multirow[t]{4}{*}{$43^{\circ} \mathrm{C} / 72 \mathrm{~h}$} & 1 & $7,0 \mathrm{~b}$ & $15,0 \mathrm{a}$ & $0,0 b$ & $5,0 \mathrm{a}$ & $2,0 \mathrm{a}$ & $2,0 \mathrm{a}$ \\
\hline & 2 & $13,0 \mathrm{a}$ & $13,0 \mathrm{a}$ & $0,0 \mathrm{~b}$ & $8,0 \mathrm{a}$ & $2,0 \mathrm{a}$ & $0,0 \mathrm{a}$ \\
\hline & 3 & $3,0 \mathrm{~b}$ & $10,0 \mathrm{a}$ & $1,0 \mathrm{~b}$ & $0,0 \mathrm{a}$ & $0,0 \mathrm{a}$ & $0,0 \mathrm{a}$ \\
\hline & 4 & $6,0 \mathrm{ab}$ & $5,0 \mathrm{~b}$ & $4,0 \mathrm{a}$ & $1,0 \mathrm{a}$ & $2,0 \mathrm{a}$ & $1,0 \mathrm{a}$ \\
\hline
\end{tabular}

${ }^{(1)}$ Médias seguidas pela mesma letra na coluna não diferem entre si pelo teste de Tukey a $5 \%$ de probabilidade. 
Tabela 5. Dados médios, em porcentagem, de fungos encontrados nas sementes e nas plântulas de amendoim provenientes de quatro lotes, com $15 \%$ e $20 \%$ de teor de água, a $40^{\circ} \mathrm{C} \mathrm{e} 45^{\circ} \mathrm{C}$, por ocasião da exposição ao teste de deterioração controlada ${ }^{(1)}$.

\begin{tabular}{|c|c|c|c|c|c|c|c|c|c|c|c|c|}
\hline \multirow[t]{2}{*}{ Lotes } & \multicolumn{3}{|c|}{$40^{\circ} \mathrm{C} / 15 \%$} & \multicolumn{3}{|c|}{$40^{\circ} \mathrm{C} / 20 \%$} & \multicolumn{3}{|c|}{$45^{\circ} \mathrm{C} / 15 \%$} & \multicolumn{3}{|c|}{$45^{\circ} \mathrm{C} / 20 \%$} \\
\hline & $\begin{array}{c}\text { Rhizopus } \\
\text { spp. }\end{array}$ & $\begin{array}{c}\text { Fusarium } \\
\text { spp. }\end{array}$ & $\begin{array}{c}\text { Aspergillus } \\
\text { spp. }\end{array}$ & $\begin{array}{c}\text { Rhizopus } \\
\text { spp. }\end{array}$ & $\begin{array}{c}\text { Fusarium } \\
\text { spp. }\end{array}$ & $\begin{array}{c}\text { Aspergillus } \\
\text { spp. }\end{array}$ & $\begin{array}{c}\text { Rhizopus } \\
\text { spp. }\end{array}$ & $\begin{array}{c}\text { Fusarium } \\
\text { spp. }\end{array}$ & $\begin{array}{c}\text { Aspergillus } \\
\text { spp. }\end{array}$ & $\begin{array}{c}\text { Rhizopus } \\
\text { spp. }\end{array}$ & $\begin{array}{c}\text { Fusarium } \\
\text { spp. }\end{array}$ & $\begin{array}{c}\text { Aspergillus } \\
\text { spp. }\end{array}$ \\
\hline 1 & $3,0 \mathrm{~b}$ & $0,0 \mathrm{a}$ & $1,0 \mathrm{~b}$ & $3,0 \mathrm{~b}$ & $2,0 \mathrm{a}$ & $0,0 \mathrm{a}$ & $11,0 \mathrm{a}$ & $0,0 \mathrm{a}$ & $0,0 \mathrm{a}$ & $10,0 b$ & $0,0 \mathrm{~b}$ & $1,0 \mathrm{a}$ \\
\hline 2 & $20,0 \mathrm{a}$ & $0,0 \mathrm{a}$ & $1,0 \mathrm{~b}$ & $45,0 \mathrm{a}$ & $2,0 \mathrm{a}$ & $0,0 \mathrm{a}$ & $9,0 \mathrm{ab}$ & $0,0 \mathrm{a}$ & $0,0 \mathrm{a}$ & $41,0 \mathrm{a}$ & $14,0 \mathrm{a}$ & $0,0 \mathrm{a}$ \\
\hline 3 & $0,0 \mathrm{~b}$ & $1,0 \mathrm{a}$ & $14,0 \mathrm{a}$ & $2,0 \mathrm{~b}$ & $3,0 \mathrm{a}$ & $3,0 \mathrm{a}$ & $5,0 \mathrm{~b}$ & $0,0 \mathrm{a}$ & $0,0 \mathrm{a}$ & $9,0 \mathrm{~b}$ & $0,0 \mathrm{~b}$ & $1,0 \mathrm{a}$ \\
\hline 4 & $32,0 \mathrm{a}$ & $4,0 \mathrm{a}$ & $0,0 \mathrm{~b}$ & $23,0 \mathrm{ab}$ & $2,0 \mathrm{a}$ & $0,0 \mathrm{a}$ & $16,0 \mathrm{a}$ & $5,0 \mathrm{a}$ & $0,0 \mathrm{a}$ & $21,0 \mathrm{ab}$ & $10,0 \mathrm{a}$ & $0,0 \mathrm{a}$ \\
\hline
\end{tabular}

${ }^{(1)}$ Médias seguidas pela mesma letra na coluna não diferem entre si pelo teste de Tukey a $5 \%$ de probabilidade.

Tabela 6. Coeficiente de correlação simples entre os resultados de deterioração controlada em relação aos de envelhecimento acelerado e da condutividade elétrica (CE), por ocasião da avaliação ao quinto e ao décimo dia ${ }^{(1)}$.

\begin{tabular}{|c|c|c|c|c|c|c|c|c|c|c|}
\hline \multirow[t]{3}{*}{ Avaliações } & \multirow{3}{*}{$\begin{array}{c}\text { Germinação } \\
(\%)\end{array}$} & \multirow{3}{*}{$\begin{array}{c}\mathrm{CE} \\
\left(\mu \mathrm{S} \mathrm{cm}^{-1} \mathrm{~g}^{-1}\right)\end{array}$} & \multicolumn{8}{|c|}{ Envelhecimento acelerado } \\
\hline & & & \multicolumn{4}{|c|}{ Com solução salina } & \multicolumn{4}{|c|}{ Sem solução salina } \\
\hline & & & $42^{\circ} \mathrm{C} / 48 \mathrm{~h}$ & $42^{\circ} \mathrm{C} / 72 \mathrm{~h}$ & $43^{\circ} \mathrm{C} / 48 \mathrm{~h}$ & $43^{\circ} \mathrm{C} / 72 \mathrm{~h}$ & $42^{\circ} \mathrm{C} / 48 \mathrm{~h}$ & $42^{\circ} \mathrm{C} / 72 \mathrm{~h}$ & $43^{\circ} \mathrm{C} / 48 \mathrm{~h}$ & $43^{\circ} \mathrm{C} / 72 \mathrm{~h}$ \\
\hline & & & \multicolumn{8}{|c|}{$5^{\circ}$ dia } \\
\hline $40^{\circ} \mathrm{C} / 15 \%$ & $0,983 * *$ & $-0,795^{\mathrm{ns}}$ & $0,951 *$ & $0,764^{\mathrm{ns}}$ & $0,994 * *$ & $0,631^{\mathrm{ns}}$ & $0,830^{\mathrm{ns}}$ & $0,501^{\mathrm{ns}}$ & $0,938 *$ & $0,542^{\mathrm{ns}}$ \\
\hline $40^{\circ} \mathrm{C} / 20 \%$ & $0,238^{\mathrm{ns}}$ & $-0,611^{\mathrm{ns}}$ & $-0,141^{\mathrm{ns}}$ & $0,664^{\mathrm{ns}}$ & $0,008^{\mathrm{ns}}$ & $-0,734^{\mathrm{ns}}$ & $0,194^{\mathrm{ns}}$ & $0,826^{\mathrm{ns}}$ & $-0,296^{\mathrm{ns}}$ & $0,159^{\text {ns }}$ \\
\hline $45^{\circ} \mathrm{C} / 15 \%$ & $0,920 *$ & $-0,990 * *$ & $0,652^{\mathrm{ns}}$ & $0,991 * *$ & $0,820^{\mathrm{ns}}$ & $0,130^{\mathrm{ns}}$ & $0,835^{\mathrm{ns}}$ & $0,884^{\mathrm{ns}}$ & $0,602^{\mathrm{ns}}$ & $0,439^{\text {ns }}$ \\
\hline $45^{\circ} \mathrm{C} / 20 \%$ & $0,819^{\mathrm{ns}}$ & $-0,487^{\mathrm{ns}}$ & $0,991 * *$ & $0,469^{\mathrm{ns}}$ & $0,831^{\text {ns }}$ & $0,680^{\text {ns }}$ & $0,451^{\mathrm{ns}}$ & $0,130^{\mathrm{ns}}$ & $0,886^{\mathrm{ns}}$ & $0,821^{\mathrm{ns}}$ \\
\hline Germinação (\%) & - & - & $0,880^{\mathrm{ns}}$ & $0,864^{\mathrm{ns}}$ & $0,971 *$ & $0,490^{\mathrm{ns}}$ & $0,846^{\mathrm{ns}}$ & $0,637^{\mathrm{ns}}$ & $0,862^{\text {ns }}$ & $0,558^{\mathrm{ns}}$ \\
\hline \multirow[t]{2}{*}{$\mathrm{CE}\left(\mu \mathrm{S} \mathrm{cm} \mathrm{c}^{-1} \mathrm{~g}^{-1}\right)$} & - & - & $0,574^{\mathrm{ns}}$ & $-0,996 * *$ & $-0,786^{\mathrm{ns}}$ & $-0,087^{\mathrm{ns}}$ & $-0,855^{\text {ns }}$ & $-0,922 *$ & $-0,550^{\mathrm{ns}}$ & $0,341^{\mathrm{ns}}$ \\
\hline & & & \multicolumn{8}{|c|}{$10 \circ$ dia } \\
\hline $40^{\circ} \mathrm{C} / 15 \%$ & $0,302^{\mathrm{ns}}$ & $-0,146^{\mathrm{ns}}$ & $-0,072^{\mathrm{ns}}$ & $0,260^{\mathrm{ns}}$ & $0,425^{\mathrm{ns}}$ & $0,227^{\mathrm{ns}}$ & $0,651^{\mathrm{ns}}$ & $-0,138^{\mathrm{ns}}$ & $0,674^{\mathrm{ns}}$ & $0,524^{\mathrm{ns}}$ \\
\hline $40^{\circ} \mathrm{C} / 20 \%$ & $-0,721^{\mathrm{ns}}$ & $-0,828^{\mathrm{ns}}$ & $-0,615^{\mathrm{ns}}$ & $-0,916^{*}$ & $-0,208^{\mathrm{ns}}$ & $-0,832^{\mathrm{ns}}$ & $-0,972 *$ & $-0,135^{\mathrm{ns}}$ & $-0,582^{\mathrm{ns}}$ & $-0,810^{\mathrm{ns}}$ \\
\hline $45^{\circ} \mathrm{C} / 15 \%$ & $0,972 *$ & $-0,946^{*}$ & $0,895^{\mathrm{ns}}$ & $0,986 * *$ & $0,530^{\mathrm{ns}}$ & $0,882^{\text {ns }}$ & $0,929 *$ & $-0,276^{\mathrm{ns}}$ & $0,755^{\mathrm{ns}}$ & $0,967 *$ \\
\hline $45^{\circ} \mathrm{C} / 20 \%$ & $0,178^{\mathrm{ns}}$ & $-0,298^{\mathrm{ns}}$ & $-0,152^{\mathrm{ns}}$ & $-0,452^{\mathrm{ns}}$ & $0,843^{\mathrm{ns}}$ & $-0,152^{\mathrm{ns}}$ & $-0,040^{\mathrm{ns}}$ & $-0,901 *$ & $0,624^{\mathrm{ns}}$ & $0,236^{\mathrm{ns}}$ \\
\hline Germinação (\%) & - & $-0,885^{\text {ns }}$ & $0,906 *$ & $0,765^{\text {ns }}$ & $0,679^{\text {ns }}$ & $0,944 *$ & $0,841^{\mathrm{ns}}$ & $-0,479^{\text {ns }}$ & $0,817^{\mathrm{ns}}$ & $0,970^{\mathrm{ns}}$ \\
\hline $\mathrm{CE}\left(\mu \mathrm{S} \mathrm{cm} \mathrm{cm}^{-1}\right)$ & - & - & $-0,944 *$ & $-0,961 *$ & $-0,260^{\mathrm{ns}}$ & $-0,988^{*}$ & $-0,845^{\mathrm{ns}}$ & $-0,337^{\mathrm{ns}}$ & $-0,889^{\mathrm{ns}}$ & $-0,834^{\mathrm{ns}}$ \\
\hline
\end{tabular}

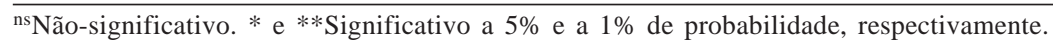

em feijão, verificaram que o tratamento de deterioração controlada com sementes com teor de água inicial de $20 \%$, por 48 horas, sob temperatura de $45^{\circ} \mathrm{C}$, apresenta alta correlação com o teste de envelhecimento acelerado, revelando as diferenças entre o potencial fisiológico de sementes, assim como também constatado na soja, por Rossetto \& Marcos Filho (1995) e Marcos Filho et al. (2001).

\section{Conclusões}

1. No teste de envelhecimento acelerado com solução salina, o período de 72 horas a $42^{\circ} \mathrm{C}$ é suficiente para avaliar o potencial fisiológico das sementes de amendoim.

2. No teste de deterioração controlada, a combinação de $15 \%$ de teor de água nas sementes e 48 horas em banho-maria a $45^{\circ} \mathrm{C}$ é eficiente para detectar diferenças de vigor entre os lotes.

\section{Agradecimento}

Ao CNPq pela concessão de bolsa aos autores.

\section{Referências}

BHERING, M.C.; DIAS, D.C.F.; BARROS, D.I.; DIAS, L.A.S.; TOKUHISA, D. Avaliação do vigor de sementes de melancia (Citrullus lunatus Schrad.) pelo teste de envelhecimento acelerado. Revista Brasileira de Sementes, v.25, p.1-6, 2003.

BRASIL. Ministério da Agricultura e Reforma Agrária. Regras para análise de sementes. Brasília: SNAD; DNPV;CLAV, 1992. 365p. COMISSÃO ESTADUAL DE SEMENTES E MUDAS (São Paulo, $\mathrm{SP}$ ). Padrões de sementes para a safra 99/2000. Campinas: Secretaria da Agricultura do Estado de São Paulo, 1999. 1p.

FREITAS, R.F. Fungos associados a grãos de café (Coffea arabica $\mathrm{L}$.) beneficiado de diversos municípios região Sul de Minas Gerais. 2000. 95p. Dissertação (Mestrado) - Universidade Federal de Lavras, Lavras. 
ITO, M.F.; BACCHI, L.M.A.; MARINGONI, A.C.; MENTEM, J.O.M. Comparação de métodos para detecção de Aspergillus spp. e Penicillium spp. em sementes de amendoim (Arachis hypogaea). Summa Phytopathologica, v.18, p.262-268, 1992.

JIANHUA, Z.; McDONALD, N.B. The saturated salt accelerated aging test for small-seeded crops. Seed Science and Technology, v.25, p.123-131, 1996.

MARCOS FILHO, J. Teste de envelhecimento acelerado. In: KRZYZANOWSKI, F.C.; VIEIRA, R.D.; FRANÇA NETO, J.B. (Ed.). Vigor de sementes: conceitos e testes. Londrina: Abrates, 1999. p.1-24.

MARCOS FILHO, J.; NOVEMBRE, A.D.C.; CHAMA, H.M.C.P. Testes de envelhecimento acelerado e de deterioração controlada para avaliação do vigor de sementes de soja. Scientia Agricola, v.58, p.421-426, 2001.

NAKAGAWA, J. Testes de vigor baseados no desempenho das plântulas. In: KRZYZANOWSKI, F.C.; VIEIRA, R.D.; FRANÇA NETO, J.B. (Ed.). Vigor de sementes: conceitos e testes. Londrina: Abrates, 1999. cap.2, p.1-24.

NOVEMBRE, A.D.L.C.; MARCOS FILHO, J. Tratamento com fungicida e conservação de sementes de feijoeiro. Revista Brasileira de Sementes, v.13, p.105-113, 1991.

PADILHA, L.; VIEIRA, M.G.G.C.; VON PINHO, E.V.R.; CARVALHO, M.L.M. Relação entre o teste de deterioração controlada e o desempenho de sementes de milho em diferentes condições de estresse. Revista Brasileira de Sementes, v.23, p.198204, 2001.

PANOBIANCO, M.; MARCOS FILHO, J. Envelhecimento acelerado e deterioração controlada em sementes de tomate. Scientia Agricola, v.58, p.525-531, 2001.

POWELL, A.A. The controlled deterioration test. In: VENTER, H.A. van de (Ed.). Seed vigour testing seminar. Copenhagen: ISTA, 1995. p.73-87.

RODO, A.B.; PANOBIANCO, M.; MARCOS FILHO, J. Metodologia alternativa do teste de envelhecimento acelerado para sementes de cenoura. Scientia Agricola, v.57, p.289-292, 2000.

ROSSETTO, C.A.V.; ARAÚJO, A.E.S.; LIMA, T.M. Avaliação da aplicação de fungicida às sementes de amendoim antes do envelhecimento acelerado. Revista Brasileira de Sementes, v.25, p.101-107, 2003.

ROSSETTO, C.A.V.; FERNANDEZ, E.M.; MARCOS FILHO, J. Metodologias de ajuste do grau de umidade e comportamento das sementes de soja no teste de germinação. Revista Brasileira de Sementes, v.17, p.171-178, 1995.

ROSSETTO, C.A.V.; MARCOS FILHO, J. Comparação entre os métodos de envelhecimento acelerado e de deterioração controlada para avaliação da qualidade fisiológica de sementes de soja. Scientia Agricola, v.52, p.123-131, 1995.

SANTOS, C.M.R.; MENEZES, N.L.; VILLELA, F.A. Teste de deterioração controlada para avaliação do vigor de sementes de feijão. Revista Brasileira de Sementes, v.25, p.28-35, 2003.

SILVA, M.A.D. da; SILVA, W.R. da. Comportamento de fungos e de sementes de feijoeiro durante o teste de envelhecimento artificial. Pesquisa Agropecuária Brasileira, v.35, p.599-608, 2000.

SINGH, K.; FRISVAD, J.C.; THRANE, U.; MATHUR, S.B. An illustrated manual on identification of some seed-borne Aspergilli, Fusaria, Penicillia and their mycotoxins. Hellerup: Danish Government Institute of Seed Pathology for Developing Countries, 1991. 133p.

TEKRONY, D.M. Accelerated aging test. Journal of Seed Technology, v.17, p.110-120, 1993.

TILDEN, R.L.; WEST, S.H. Reversal of the effects of ageing in soybean seeds. Plant Physiology, v.77, p.584-586, 1985.

USBERTI, R.; AMARAL, H.M. Fungicide dressing timing, seed size, seed origin and fungal incidence effects on groundnut (Arachis hypogaea L.) storability. Seed Science and Technology, v.27, p.699-706, 1999.

VANZOLINI, S.; NAKAGAWA, J. Teste de condutividade elétrica em genótipos de amendoim. Revista Brasileira de Sementes, v.20, p.178-183, 1998.

Recebido em 16 de fevereiro de 2004 e aprovado em 6 de maio de 2004 